\title{
The clinical role of multimodality imaging in the detection of prostate cancer recurrence after radical prostatectomy and radiation therapy: past, present, and future
}

\author{
Francesco Paparo', Michela Massollo ${ }^{2}$, Ludovica Rollandi ${ }^{3}$, Arnoldo Piccardo ${ }^{2}$, Filippo Grillo Ruggieri ${ }^{4}$ and \\ Gian Andrea Rollandi ${ }^{1}$ \\ ${ }^{1}$ Radiology Unit, Department of Diagnostic Imaging, E O Galliera Hospital, Mura delle Cappuccine 14, 16128 Genoa, Italy \\ ${ }^{2}$ Nuclear Medicine Unit, Department of Diagnostic Imaging, E O Galliera Hospital, Mura delle Cappuccine 14, 16128 Genoa, Italy \\ ${ }^{3}$ Klinikum Augsburg Radiologie, Stelingstrasse 2, 86156 Augsburg, Germany \\ ${ }^{4}$ Radiotherapy Unit, Department of Diagnostic Imaging, E O Galliera Hospital, Mura delle Cappuccine 14, 16128 Genoa, Italy
}

Correspondence to: Francesco Paparo. Email: francesco.paparo@galliera.it

\begin{abstract}
Detection of the recurrence sites in prostate cancer (PCa) patients affected by biochemical recurrence after radical prostatectomy (RP) and radiation therapy $(\mathrm{RT})$ is still a challenge for clinicians, nuclear medicine physicians, and radiologists. In the era of personalised and precision care, this task requires the integration, amalgamation, and combined analysis of clinical and imaging data from multiple sources. At present, multiparametric Magnetic Resonance Imaging ( $\mathrm{mpMRI}$ ) and choline-positron emission tomography (PET) are giving encouraging results; their combination allows the effective detection of local, lymph nodal, and skeletal recurrences at low PSA levels. Future diagnostic perspectives include the clinical implementation of PET/MRI scanners, multimodal fusion imaging platforms for retrospective co-registration of PET and MR images, real-time transrectal ultrasound/mpMRI fusion imaging, and novel organ-specific PET radiotracers.
\end{abstract}

Keywords: prostate cancer, magnetic resonance imaging, choline, positron emission tomography, fusion imaging

Published: 04/09/2015

Received: 30/03/2015

ecancer 2015, 9:570 DOI: 10.3332/ecancer.2015.570

Copyright: (c) the authors; licensee ecancermedicalscience. This is an Open Access article distributed under the terms of the Creative Commons Attribution License (http://creativecommons.org/licenses/by/3.0), which permits unrestricted use, distribution, and reproduction in any medium, provided the original work is properly cited. 


\section{Background and discussions}

Measurement of Prostate Specific Antigen (PSA) in serum is a cornerstone in the monitoring of asymptomatic PCa cancer patients after curative treatment, including both RP and RT. A rapidly increasing PSA level (i.e., high PSA velocity and short PSA doubling time) is usually related to the presence of distant metastases (i.e., skeletal or lymph nodal), while a slow and progressive increase in serum PSA concentration is often because of local disease recurrence [1].

In patients with biochemical recurrence, imaging plays a key role in the identification of the site of PCa recurrence, and imaging documentation is often required for establishing an appropriate second-line treatment [2, 3]. A palpable nodule at digital rectal examination (DRE) accompanied by a rising PSA level are signs of local disease recurrence [3]. However, DRE and transrectal ultrasound (TRUS) are neither sensitive nor specific in detecting local recurrences after RP and RT [4]. After both RP and RT, advanced multimodality imaging can be successfully employed to provide a detailed documentation of the PCa relapse, including local, lymph nodal, and skeletal recurrences, and also for establishing an appropriate second-line treatment.

\section{The past: TRUS, bone scintigraphy and abdominopelvic CT}

\section{Transrectal ultrasound (TRUS) and TRUS-guided biopsy}

TRUS has a low diagnostic accuracy in the detection of local PCa recurrence after RP because of several limitations, which mainly include isoechoic lesions, post-treatment changes of the normal pelvic anatomy, and small volume recurrences. Locally recurrent PCa is hypoechoic in about $65 \%$ of cases, while about $30 \%$ of local recurrences have the same echogenicity of the vesicourethral anastomosis $[5,6]$. When TRUS enables the identification of suspected areas in the prostatic fossa, the use of TRUS-guidance for targeting the biopsy procedure increases the rate of positive sampling [7]. However, the ability of TRUS to detect a local recurrence strongly depends on PSA levels. In particular, TRUS is able to detect every biopsy-proven local recurrence only with PSA levels $>2.0 \mathrm{ng} / \mathrm{mL}$ [6]; at lower PSA levels the clinical use of TRUS is questionable. The sensitivity of anastomotic biopsies under TRUS guidance is variable, with values ranging from $40 \%$ to $71 \%$ for PSA levels $>1 \mathrm{ng} / \mathrm{mL}$, but it drops down to $14 \%-45 \%$ for PSA levels $<1 \mathrm{ng} / \mathrm{mL}$ [2, 4]. Therefore, a TRUS-guided negative biopsy is not adequate to rule out the presence of a local recurrence. In accordance to the last guidelines of the European Urology Association [1], salvage RT is generally recommended on the basis of the evidence of biochemical recurrence without any histological proof of the local recurrence. After RT, diagnosing local recurrence is challenging because of radiation-induced fibrosis and shrinkage of the gland [2, 4]. In this setting, the reported values of sensitivity and specificity for TRUS are $49 \%$ and $57 \%$, respectively [8]. TRUS-guided biopsy after RT has the main role of confirming or rule out the presence of local PCa recurrence; the information provided by needle biopsy is also important to assess the tumour spread in the gland and is essential for planning the salvage therapy (cryosurgery or prostatectomy) $[9,10]$. After RT, prostate biopsy is not considered the reference standard for assessing treatment efficacy, but is an independent predictor of outcome [10].

\section{Bone scintigraphy and computed tomography}

Until recently, bone scintigraphy with Technetium-99m medronic acid (Tc-99m MDP) and contrast-enhanced abdominopelvic computed tomography (ceCT) have been considered the main diagnostic tools to detect skeletal and lymph nodal metastases. However, the diagnostic yield of these well-established imaging techniques is not satisfactory in patients with a serum PSA level $<10 \mathrm{ng} / \mathrm{mL}$ or a low PSA velocity $(<0.5 \mathrm{ng} / \mathrm{mL} / \mathrm{month})$ [11] (Figure 1). For example, only $12.5 \%$ of patients with biochemical failure after RP have a positive ceCT [11], and in a series of 132 men affected by biochemical failure, the mean PSA level and PSA velocity associated with a positive ceCT were $27.4 \mathrm{ng} / \mathrm{mL}$ and $1.8 \mathrm{ng} / \mathrm{mL} / \mathrm{month}$ respectively [12]. By means of newer imaging techniques, systemic disease can be identified when PSA levels are significantly lower $[2,13]$.

\section{The present: multiparametric MRI and choline-PET}

\section{Multiparametric MRI}

Currently, the most promising diagnostic modalities for evaluating PCa patients with biochemical recurrence include mpMRI and PET/CT with radio-labeled choline derivatives. With regard to MRI, the detection of local recurrence after both RP and RT is not easy by using the 
information provided by morphological imaging alone (i.e., T2-weighted sequences) [14]. In mpMRI, morphological T2-weighted sequences are combined with functional techniques, including diffusion weighted imaging (DWI), dynamic contrast-enhanced (DCE) perfusion imaging, and spectroscopy [2,3]. In the detection of local relapse after RP, T2-weighted imaging is characterised by low sensitivity (48\%-61\%) and specificity $(52 \%-82 \%)$ [15, 16]; false-positive diagnoses may occur when postoperative scarring assumes a nodular appearance, mimicking recurrence. DCE-MRI is a valuable functional technique for distinguishing PCa recurrence from fibrosis in the prostatectomy fossa and remnants of normal prostatic tissue. When assessed in combination with T2-weighted imaging, DCE-MRI is particularly accurate in detecting PCa recurrence after RP with sensitivity and specificity values of $79 \%-88 \%$ and $89 \%-100 \%$ respectively [15-18] (Figure 2). After RT, radiation-induced morphological changes in the prostate include inflammation, glandular atrophy, fibrosis, and shrinkage [3, 4]. On T2-weighted images, radiation-induced changes often cause a diffusely reduced signal intensity of the gland parenchyma, more evident in the peripheral zone, with consequent loss of the physiological zonal anatomy (Figure 3). Local PCa recurrences are characterised by low-signal intensity on T2-weighted images; therefore, they are often difficult to distinguish from the surrounding irradiated prostate tissue, which has a comparable signal intensity $[19,20]$. Given the low sensitivity $(26 \%-44 \%)$ and moderate specificity $(64 \%-86 \%)$ of T2-weighted imaging in detecting local recurrence, the use of additional functional techniques is mandatory to achieve a precise diagnosis [21-23].

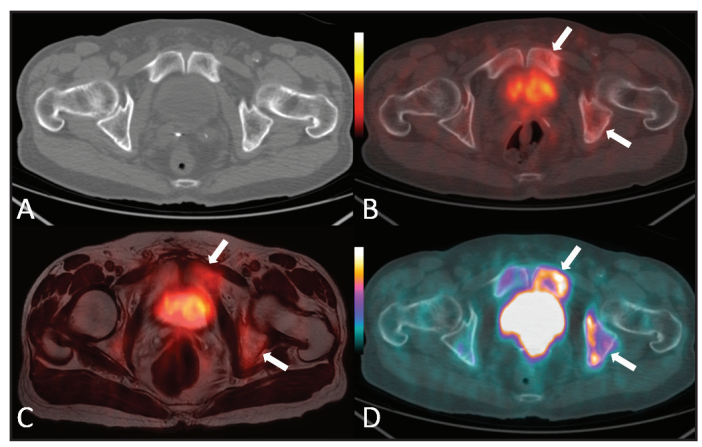

Figure 1. An 80-year-old man with bone metastases involving the left ischiopubic branch. The bone lesions are not detectable on the axial CT image with bone window (A) The ${ }^{18} \mathrm{~F}$-choline-PET/CT (B) and the ${ }^{18} \mathrm{~F}$-choline-PET/MRI fused (C) axial images show areas of moderate tracer accumulation in the left ischiopubic branch (arrows). The presence of metastatic bone involvement was further confirmed by means of ${ }^{18} \mathrm{~F}$-fluoride-PET/CT scan (D) Images obtained with Quanta Prostate, Camelot Biomedical Systems s.r.l., Genoa, Italy.

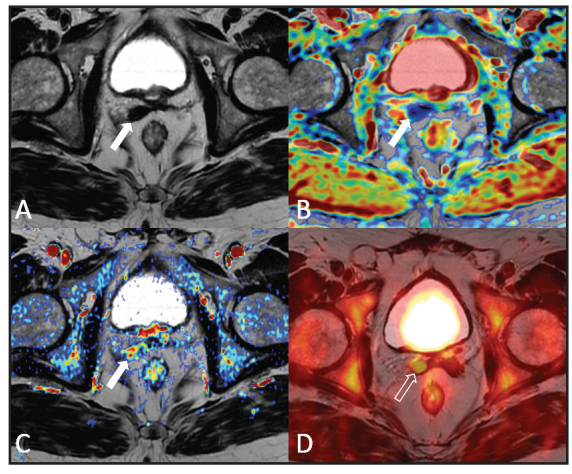

Figure 2. A 73-year-old patient who underwent radical prostatectomy 40 months earlier for prostate cancer Gleason 7 (3 + 4). Current PSA value of $2.2 \mathrm{ng} / \mathrm{mL}$. The T2-weighted axial image (A) shows a hypointense nodular thickening of the remnant of the right seminal vesicle (arrow), which is characterised by restricted water diffusion on the apparent diffusion coefficient (ADC) map (B) and is hypervascular on the perfusion map obtained from the DCE-MRI acquisition (C) The ${ }^{18} \mathrm{~F}$-choline-PET/MRI fused axial image (D) shows a focal accumulation of the tracer in correspondence to the remnant of the right seminal vesicle (void arrow), which confirmed the diagnosis of local relapse. Images obtained with Quanta Prostate, Camelot Biomedical Systems s.r.I., Genoa, Italy. 


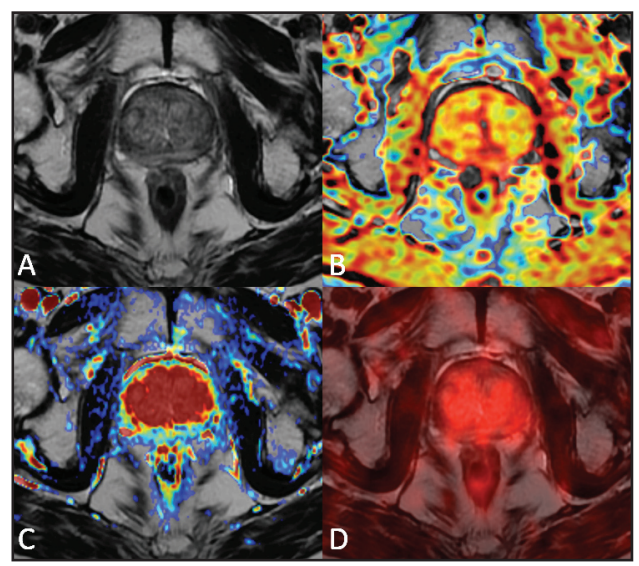

Figure 3. A 75-year-old patient treated with external beam radiation therapy. The T2-weighted axial image (A) shows diffuse low signal intensity of the prostate gland with loss of the normal zonal anatomy. The ADC map obtained from the diffusion-weighted sequence does not show any significant focus of restricted water diffusion $(B)$ The transition zone is homogeneously hypervascular on the perfusion map obtained from the DCE-MRI acquisition (C) The ${ }^{18} \mathrm{~F}$-choline-PET/MRI fused axial image (D) does not show any focal accumulation of the tracer in the prostate parenchyma. This case underscores that diffusely reduced signal intensity of the prostate parenchyma after RT with loss of zonal anatomy is not necessarily a sign of local recurrence. Images obtained with Quanta Prostate, Camelot Biomedical Systems s.r.I., Genoa, Italy.

\section{Choline-PET/CT}

Choline-PET/CT is a whole-body molecular imaging technique that is able to show the metabolic activity of phospholipid turnover within cellular membranes.

In a recent comparative study, the patient-based sensitivity, specificity, and accuracy of choline-PET/CT in diagnosing local recurrence were $54.1 \%, 92.3 \%$, and $65.5 \%$ respectively, whereas those of $\mathrm{mpMRI}$ were $88.5 \%, 84.6 \%$, and $87.4 \%$ [24]. Despite some limitations in detecting local recurrences, choline-PET/CT has the great advantage of detecting lymph-node metastases when they are not discernible at morphological imaging (i.e., ceCT and MRI) $[2,12,13]$. This peculiar feature of choline-PET/CT has an important clinical value, since up to $80 \%$ of metastatic lymph nodes in PCa have a short-axis diameter smaller than $7 \mathrm{~mm}$ [25], which produces a lot of false negatives at morphological imaging. Indeed, a meta-analysis by Hövels et al [26] reported a pooled sensitivity of $42 \%$ and $39 \%$ for CT and MRI respectively, and pooled specificity of $82 \%$ for both imaging techniques. Some previous studies evaluated the diagnostic yield of choline-PET/CT in lymph node staging in patients with biochemical failure after primary treatment, using lymph node dissection as standard of reference [27-29]. Scattoni et al reported a sensitivity of $64 \%$, a specificity of $90 \%$, a positive predictive value of $86 \%$, and a negative predictive value of $72 \%$ [27]; inadequate detection of lymph node micrometastases was considered the main explanation for the low sensitivity of PET/CT. By converse, Rinnab et al found a 100\% sensitivity for choline-PET/CT, but its positive predictive value was only 53\% [28]. Another study found a high false positive rate with $30 \%$ of patients with pelvic lymph node metastases on choline-PET/CT having no pathological confirmation [29]. In accordance to the results of a meta-analysis by Umbehr et al, the pooled sensitivity, specificity, and the diagnostic odds ratio of choline-PET/CT were $85 \%, 88 \%$, and 41.4 , respectively, on a per-patient basis [30].

When considering skeletal metastases, both choline-PET/CT and mpMRI show excellent diagnostic performances [13, 14, 31]. Occasionally, choline-PET/CT may give false negatives when assessing skeletal metastases with dense sclerosis on CT; indeed choline activity tends to reduce in relation to increasing lesion sclerosis $[13,20]$. By converse, sclerotic metastases are not a limitation for mpMRI by means of DWI and short tau inversion recovery (STIR) sequences [2, 3, 32]. Hence summarising, pelvic mpMRI is superior to choline-PET in depicting local relapse after RP and RT, whereas choline-PET/CT is more accurate than mpMRI in detecting lymph nodal metastatic disease; both techniques are excellent in the assessment of skeletal metastases [2, 3, 8]. 


\section{The future: multimodality fusion imaging and novel PET-radiotracers}

\section{Multimodality fusion imaging}

The combined and synchronised assessment of spatially aligned mpMRI and PET images by means of multimodal fusion imaging is currently giving encouraging and promising results [14]. Multimodality fusion imaging can be performed by means of simultaneous acquisition, which requires integrated PET/MRI scanners [33,34], or retrospective co-registration of previously acquired PET and MRI datasets by means of dedicated software platforms [2, 14, 35] (Figure 4). A recent study by Piccardo et al has confirmed high detection rates for both modalities (i.e., $76 \%$ for $18-$ fluorine (18F)-choline-PET/CT and $81 \%$ for mpMRI) in patients affected by biochemical recurrence after RT [14]. Multimodality retrospective fusion imaging between choline-PET/CT and mpMRI (fused 18F-choline-PET/mpMRI) yielded an even better detection rate (i.e., 86\%). These results underline that multimodal co-registration and combined interpretation are more valuable than the separate assessment of different imaging techniques [2, 14] (Figure 5). After RP and RT, the synchronous assessment of DCE-MRI and dynamic perfusion curves with the metabolic information provided by choline-PET may be beneficial to reach a correct diagnosis. Trimodal real-time fusion imaging involving TRUS, mpMRI, and choline-PET can be performed by means of dedicated software platforms implemented on high-end multipurpose ultrasound systems [2, 35, 36]. It seems to be an excellent approach to obtain histological proof of PCa recurrence after both RP and RT [2] (Figure 6). In these patients, the main salvage option is RP, but radiation-induced changes result in a higher risk of urinary incontinence and rectal injury than in the primary setting. Therefore, given the morbidity of salvage options, it is beneficial to obtain histological proof of the local recurrence before treating the patient $[1,2,37]$.

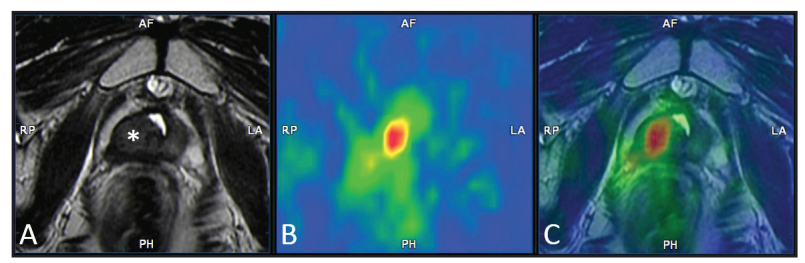

Figure 4. Example of bimodal ${ }^{18} \mathrm{~F}$-choline-PET/MRI fusion imaging in a 78 -year-old patient who had undergone external beam radiation therapy 24 months earlier for prostate cancer Gleason $7(3+4)$. Signs of a previous TURP are also present. The T2-weighted axial image (A) shows a hypointense nodular thickening of the right lobe of the gland (asterisk). The 18F-choline-PET axial image (B) shows a focal accumulation of the tracer adjacent to the mid-line of the pelvis. The ${ }^{18} \mathrm{~F}$-choline-PET/MRI fused axial image shows the precise correspondence between the MRI finding and the focal accumulation of the tracer, thus confirming the suspect of local recurrence. Images obtained with the co-registration tool of the Virtual Navigation System, Esaote Biomedica S.p.A., Genoa, Italy.

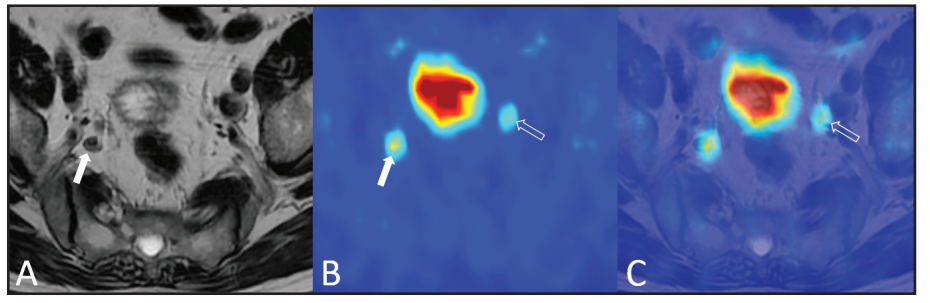

Figure 5. A 79-year-old man with bilateral hypogastric lymph node metastases. T2-weighted axial image (A) shows a right hypogastric lymphadenopathy (arrow). The ${ }^{18} \mathrm{~F}$-choline-PET axial scan (B) demonstrates two areas of focal tracer uptake (arrow and void arrow). The ${ }^{18} \mathrm{~F}$-choline-PET/MRI fused axial image (C) shows that the left focus of tracer uptake corresponded to a tiny hypogastric lymph node, which was not evident on the morphological T2-weighted image (void arrow). 


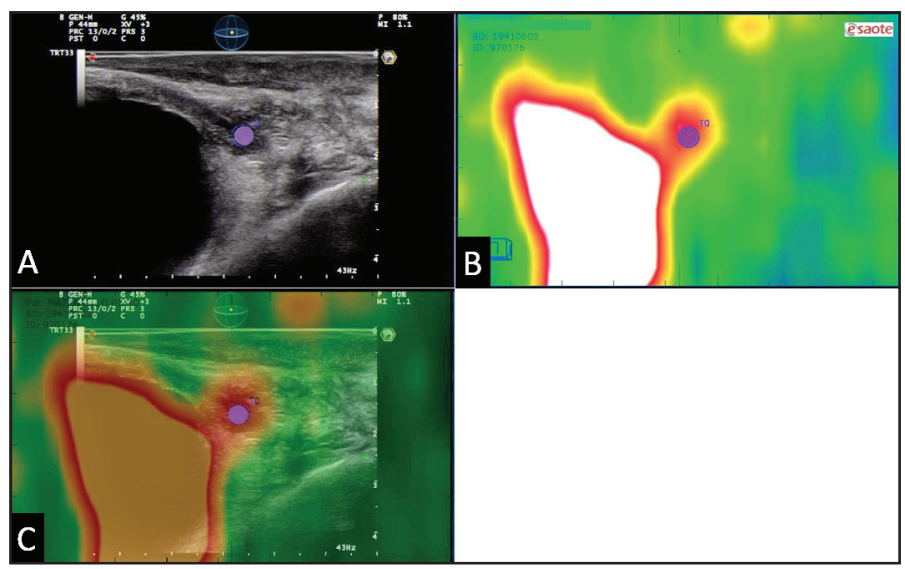

Figure 6. Example of ${ }^{18} \mathrm{~F}$-choline-PET/TRUS fusion imaging in a 72-year-old patient treated three years earlier with radical prostatectomy for prostate cancer Gleason $8(5+3)$. Current PSA value of $3.5 \mathrm{ng} / \mathrm{mL}$. The longitudinal TRUS scan (A) obtained with a biplanar (linear and sectorial) endorectal probe (TRT33, Esaote, Genoa, Italy) shows an ill-defined hypoechoic area in the retrovesical space/prostatic fossa (blue marker). On the sagittal ${ }^{18} \mathrm{~F}$-choline-PET image $(\mathrm{B})$ the round blue marker indicates the focal tracer accumulation. The fused ${ }^{18} \mathrm{~F}-\mathrm{choline-PET/TRUS}$ image (C) clearly shows the spatial correspondence between the PET-positive focus and the hypoechoic area, which was a biopsy-proven local recurrence. Images obtained with the Virtual Navigation System, Esaote Biomedica S.p.A., Genoa, Italy.

\section{Novel PET-radiotracers}

Future perspectives in the diagnosis, follow-up, and targeted treatment of PCa also encompass the novel PET radio-tracers. Extensive studies are being conducted to develop various PET imaging agents targeting specific biomarkers of PCa, including gastrin-releasing peptide receptor (GRPR) [38] and prostate-specific membrane antigen (PSMA) [39]. GRPR-binding ligands represent potential diagnostic and therapeutic agents for the targeting of GRPR-positive tumours, including PCa. In particular, the GRPR has been shown to be overexpressed in $63 \%-100 \%$ of primary PCa and in more than $50 \%$ of lymph node and bone metastases [38]. The GRPR density is 26 -fold higher in PCa than in prostate hyperplasia, thus providing substantial advantages over choline- and acetate-based radiotracers [40, 41]. PSMA is considered a highly specific prostate epithelial cell membrane antigen [42]. PSMA is expressed in other 'non-target' tissues including normal (benign) prostate epithelium, the small intestine, renal tubular cells, and salivary gland, but 100-1000 fold less than the baseline expression in PCa [43-45]. The unique functional features and the high specificity for PCa makes PSMA a theoretically perfect extracellular target for various imaging and therapeutic agents. In addition, an elevated expression of PSMA is associated with metastasis, androgen independence, and progression [46-49]. A recent study compared the diagnostic yield of ${ }^{18} \mathrm{~F}$-choline-PET/CT with that of a novel PET/CT imaging technique based on a $68-$ Gallium $\left({ }^{68} \mathrm{Ga}\right)$-labelled PSMA ligand [50]. The detection rate was significantly higher for 68Ga-PSMA PET/CT, especially at low PSA levels, and all the lesions detected by choline-PET/CT were also seen on ${ }^{68} \mathrm{Ga}$-PSMA PET/CT. This result underlines that choline metabolism is not increased in a considerable number of prostate cancers, whereas the PSMA is overexpressed in most cases. Choline-PET showed lower sensitivity in detecting lymph node metastases; a significant advantage of ${ }^{68} \mathrm{Ga}-\mathrm{PSMAPET} / \mathrm{CT}$ over choline-PET was the findings, which are typical for lymph node metastases, presented with higher contrast and excellent signal-to-background ratio [50]. In a cohort of 248 patients showing biochemical recurrence after RP, Eiber et al demonstrated that ${ }^{68} \mathrm{Ga}-\mathrm{PSMA} P E T / C T$ is able to provide detection rates $>90 \%$ at PSA levels $>1 \mathrm{ng} / \mathrm{mL}$ [51]. Most importantly, the authors found a significant number of positive findings even at lower PSA values $(<0.5 \mathrm{ng} / \mathrm{mL}) .{ }^{68} \mathrm{Ga}$-PSMA PET/CT detected lymph node metastases in 31 patients, while unenhanced CT in only one patient. Thus summarising, the main advantages of ${ }^{68} \mathrm{Ga}-\mathrm{PSMA} P E T / C T$ are the sensitive detection of lesions at low PSA levels, including small lymph nodes, bone and liver metastases, primarily because of a high radiotracer uptake and low background signal [51].

Recently, promising data on PET/CT-guided salvage lymph node dissection in patients with biochemical recurrence after RP have been reported [52, 53]. Winter et al showed that choline-PET/CT-guided salvage resection of lymph node metastases is able to provide a longterm complete biochemical remission, without the use adjuvant therapy, in certain PCa patients with biochemical recurrence after RP [52]. In this study, histology confirmed the 13 of 16 lymph nodes suspicious on PET/CT imaging [52]. The majority of patients with histologicallyconfirmed lymph node metastases showed a PSA response after lymphadenectomy; in addition, three of ten patients with a single lymph node metastasis had a complete biochemical remission (PSA $<0.01 \mathrm{ng} / \mathrm{mL}$ ). In five patients with a single lymph node metastasis, the 
serum PSA decreased at values $<0.02 \mathrm{ng} / \mathrm{mL}$ [52]. Therefore, in those patients showing disease relapse limited to lymph nodes, salvage lymphadenectomy seems to represent a valid therapeutic option after RP [53].

\section{Conclusion}

The effective detection of PCa recurrence after primary treatment requires a well-trained and experienced multidisciplinary team in order to combine the morphological, functional and metabolic information coming from different techniques and imaging modalities.

\section{References}

1. Mottet $\mathrm{N}$ et al (2011) EAU guidelines on prostate cancer. Part II: Treatment of advanced, relapsing, and castration-resistant prostate cancer Eur Urol 59(4) 572-83 Epub 2011 Jan 25 DOI: 10.1016/j.eururo.2011.01.025 PMID: 21315502

2. Paparo $\mathrm{F}$ et al (2015) Value of bimodal (18) F-choline-PET/MRI and trimodal (18) F-choline-PET/MRI/TRUS for the assessment of prostate cancer recurrence after radiation therapy and radical prostatectomy Abdom Imaging 40(6) 1772-87 [Epub ahead of print] DOI: 10.1007/s00261-014-0345-0 PMID: 25579170

3. De Visschere PJ et al (2013) Imaging treated prostate cancer Abdom Imaging 38(6) 1431-46 DOI: 10.1007/s00261-013-9998-3 PMID: 23604966

4. Martino $P$ et al (2011) Role of imaging and biopsy to assess local recurrence after definitive treatment for prostate carcinoma (surgery, radiotherapy, cryotherapy, HIFU) World J Urol 29(5) 595-605 Epub 2011 May 8 DOI: 10.1007/s00345-011-0687-y PMID: $\underline{21553276}$

5. Leventis AK, Shariat SF and Slawin KM (2001) Local recurrence after radical prostatectomy: correlation of US features with prostatic fossa biopsy findings Radiology 219(2) 432-9 DOI: 10.1148/radiology.219.2.r01ma20432 PMID: 11323468

6. Scattoni $\mathrm{V}$ et al (2003) Multiple vesico-urethral biopsies following radical prostatectomy: the predictive roles of TRUS, DRE, PSA and the pathological stage Eur Urol 44(4) 407-14 DOI: 10.1016/S0302-2838(03)00320-8 PMID: 14499673

7. Parra RO, Wolf RM and Huben RP (1990) The use of transrectal ultrasound in the detection and evaluation of local pelvic recurrences after a radical urological pelvic operation J Urol 144(3) 707-9 PMID: 2201797

8. Crook $\mathrm{J}$ et al (1993) Clinical relevance of trans-rectal ultrasound, biopsy, and serum prostate-specific antigen following external beam radiotherapy for carcinoma of the prostate Int J Radiat Oncol Biol Phys 27(1) 31-7 DOI: 10.1016/0360-3016(93)90418-U PMID: $\underline{7690016}$

9. Galosi AB, Lugnani $F$ and Muzzonigro G (2007) Salvage cryosurgery for recurrent prostate carcinoma after radiotherapy J Endourol 21(1) 1-7 DOI: 10.1089/end.2006.9999 PMID: 17263599

10. Crook J et al (2000) Postradiotherapy prostate biopsies: what do they really mean? Results for 498 patients Int J Radiat Oncol Biol Phys 48(2) 355-67 DOI: 10.1016/S0360-3016(00)00637-4 PMID: 10974448

11. Beresford MJ et al (2010) A systematic review of the role of imaging before salvage radiotherapy for post-prostatectomy biochemical recurrence Clin Oncol (R Coll Radiol) 22(1) 46-55 Epub 2009 Nov 30 DOI: 10.1016/j.clon.2009.10.015 PMID: 19948393

12. Kane CJ et al (2003) Limited value of bone scintigraphy and computed tomography in assessing biochemical failure after radical prostatectomy Urology 61(3) 607-11 DOI: 10.1016/S0090-4295(02)02411-1 PMID: 12639656

13. Kitajima K, Murphy RC and Nathan MA (2013) Choline PET/CT for imaging prostate cancer: an update Ann NuCl Med 27(7) 581-91 Epub 2013 Apr 30 DOI: 10.1007/s12149-013-0731-7 PMID: 23632880

14. Piccardo A et al (2014) Value of fused 18F-Choline-PET/MRI to evaluate prostate cancer relapse in patients showing biochemical recurrence after EBRT: preliminary results Biomed Res Int 103718 Epub 2014 Apr 30 DOI: 10.1155/2014/103718 PMID: 24877053 PMCID: $\underline{4022120}$ 
15. Cirillo $S$ et al (2009) Endorectal magnetic resonance imaging at 1.5 Tesla to assess local recurrence following radical prostatectomy using T2-weighted and contrast-enhanced imaging Eur Radiol 19(3) 761-9 Epub 2008 Sep 30 DOI: $10.1007 /$ s00330-008-1174-8 PMID: 18825386

16. Casciani $\mathrm{E}$ et al (2008) Endorectal and dynamic contrast-enhanced MRI for detection of local recurrence after radical prostatectomy AJR Am J Roentgenol 190(5) 1187-92 DOI: 10.2214/AJR.07.3032 PMID: 18430830

17. Sciarra A et al (2007) Role of dynamic contrast-enhanced magnetic resonance (MR) imaging and proton MR spectroscopic imaging in the detection of local recurrence after radical prostatectomy for prostate cancer Eur Urol 54(3) 589-600 Epub 2007 Dec 31 DOI: 10.1016/j.eururo.2007.12.034 PMID: 18226441

18. Panebianco $\mathrm{V}$ et al (2013) Prostate cancer recurrence after radical prostatectomy: the role of 3-T diffusion imaging in multiparametric magnetic resonance imaging Eur Radiol 23(6) 1745-52 Epub 2013 Feb 2 DOI: 10.1007/s00330-013-2768-3 PMID: $\underline{23377546}$

19. Pucar D et al (2005) Prostate cancer: correlation of MR imaging and MR spectroscopy with pathologic findings after radiation therapy-initial experience Radiology 236(2) 545-53 Epub 2005 Jun 21 DOI: 10.1148/radiol.2362040739 PMID: 15972335 PMCID: $\underline{2373272}$

20. Coakley FV et al (2004) Endorectal MR imaging and MR spectroscopic imaging for locally recurrent prostate cancer after external beam radiation therapy: preliminary experience Radiology 233(2) 441-8 Epub 2004 Sep 16 DOI: 10.1148/radiol.2332032086 PMID: $\underline{15375223}$

21. Kim CK et al (2010) Prostate MR imaging at $3 \mathrm{~T}$ using a phased-arrayed coil in predicting locally recurrent prostate cancer after radiation therapy: preliminary experience Abdom Imaging 35(2) 246-52 Epub 2009 Jan 7 DOI: 10.1007/s00261-008-9495-2 PMID: 19130116

22. Vargas HA et al (2012) MR imaging of treated prostate cancer Radiology 262(1) 26-42 DOI: 10.1148/radiol.11101996 PMID: $\underline{22190655}$

23. Rouvière $\mathrm{O}$ et al (2004) Recurrent prostate cancer after external beam radiotherapy: value of contrast-enhanced dynamic MRI in localizing intraprostatic tumor-correlation with biopsy findings Urology 63(5) 922-7 DOI: 10.1016/j.urology.2003.12.017 PMID: 15134982

24. Kitajima K et al (2014) Detection of recurrent prostate cancer after radical prostatectomy: comparison of 11C-choline PET/ CT with pelvic multiparametric MR imaging with endorectal coil J Nucl Med 55(2) 223-32 Epub 2014 Jan 16 DOI: 10.29671 inumed.113.123018 PMID: 24434294

25. Fortuin A et al (2013) Molecular and functional imaging for detection of lymph node metastases in prostate cancer Int J Mol Sci

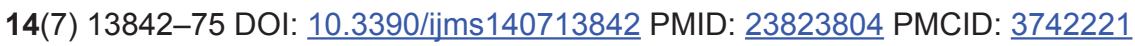

26. Hövels AM et al (2008) The diagnostic accuracy of CT and MRI in the staging of pelvic lymph nodes in patients with prostate cancer: a meta-analysis Clin Radiol 63(4) 387-95 Epub 2008 Feb 4 DOI: 10.1016/j.crad.2007.05.022 PMID: 18325358

27. Scattoni $V$ et al (2007) Detection of lymph-node metastases with integrated [11C]choline PET/CT in patients with PSA failure after radical retropubic prostatectomy: results confirmed by open pelvic-retroperitoneal lymphadenectomy Eur Urol 52(2) 423-9 Epub 2007 Mar 20 DOI: 10.1016/i.eururo.2007.03.032 PMID: 17397992

28. Rinnab L et al (2008) [11C] Choline PET/CT for targeted salvage lymph node dissection in patients with biochemical recurrence after primary curative therapy for prostate cancer. Preliminary results of a prospective study Urol Int 81(2) 191-7 Epub 2008 Aug 29 DOI: $10.1159 / 000144059$ PMID: 18758218

29. Schilling D et al (2008) Histological verification of 11C-choline-positron emission/computed tomography-positive lymph nodes in patients with biochemical failure after treatment for localized prostate cancer BJU Int 102(4) 446-51 Epub 2008 Apr 11 DOI: 10.1111/j.1464-410X.2008.07592.x PMID: 18410442 
30. Umbehr $\mathrm{MH}$ et al (2013) The role of 11C-choline and 18F-fluorocholine positron emission tomography (PET) and PET/ CT in prostate cancer: a systematic review and meta-analysis Eur Urol 64(1) 106-17 Epub 2013 Apr 19 DOI: 10.1016/j. eururo.2013.04.019 PMID: $\underline{23628493}$

31. Even-Sapir E et al (2006) The detection of bone metastases in patients with high-risk prostate cancer: 99mTc-MDP Planar bone scintigraphy, single- and multi-field-of-view SPECT, 18F-fluoride PET, and 18F-fluoride PET/CT J Nucl Med 47(2) 287-97 PMID: $\underline{16455635}$

32. Beheshti $\mathrm{M}$ et al (2009) The use of F-18 choline PET in the assessment of bone metastases in prostate cancer: correlation with morphological changes on CT Mol Imaging Biol 11(6) 446-54 Erratum in: Mol Imaging Biol 2010 Jun 12(3) 360 DOI: $10.1007 /$ s11307-009-0217-0 PMID: 19326171

33. Pichler BJ et al (2008) Positron emission tomography/magnetic resonance imaging: the next generation of multimodality imaging? Semin Nucl Med 38(3) 199-208 DOI: 10.1053/j.semnuclmed.2008.02.001 PMID: 18396179 PMCID: 2762705

34. Wetter A et al (2014) Quantitative evaluation of bone metastases from prostate cancer with simultaneous [18F] choline PET/ MRI: combined SUV and ADC analysis Ann Nucl Med 28(5) 405-10 Epub 2014 Mar 5 DOI: 10.1007/s12149-014-0825-x PMID: $\underline{24595461}$

35. Paparo $\mathrm{F}$ et al (2015) Multimodality fusion imaging in abdominal and pelvic malignancies: current applications and future perspectives Abdom Imaging [Epub ahead of print] DOI: 10.1007/s00261-015-0435-7 PMID: 25952574

36. Paparo $\mathrm{F}$ et al (2014) Advantages of percutaneous abdominal biopsy under PET-CT/ultrasound fusion imaging guidance: a pictorial essay Abdom Imaging 39(5) 1102-13 DOI: 10.1007/s00261-014-0143-8 PMID: 24777592

37. Rouvière O, Vitry T and Lyonnet D (2010) Imaging of prostate cancer local recurrences: why and how? Eur Radio/ 20(5) 1254-66 Epub 2009 Nov 17 DOI: 10.1007/s00330-009-1647-4 PMID: 19921202

38. Sancho V et al (2011) Bombesin receptor-mediated imaging and cytotoxicity: review and current status Curr Drug Deliv 8(1) 79-134 DOI: 10.2174/156720111793663624 PMID: 21034419 PMCID: 3058932

39. Osborne JR et al (2013) Prostate-specific membrane antigen-based imaging Urol Oncol 31(2) 144-54 Epub 2012 May 31 DOI: 10.1016/j.urolonc.2012.04.016 PMID: 22658884 PMCID: $\underline{3461099}$

40. Delgado Bolton RC et al (2009) [Positron emission tomography (PET) and PET-CT in renal, bladder and prostate cancer: update] Actas Urol Esp 33(1) 11-23 DOI: 10.1016/S0210-4806(09)73997-X PMID: 19462720

41. Markwalder R and Reubi JC (1999) Gastrin-releasing peptide receptors in the human prostate: relation to neoplastic transformation Cancer Res 1999 59(5) 1152-9 PMID: 10070977

42. Horoszewicz JS, Kawinski E and Murphy GP (1987) Monoclonal antibodies to a new antigenic marker in epithelial prostatic cells and serum of prostatic cancer patients Anticancer Res 7(5B) 927-35 PMID: 2449118

43. Israeli RS et al (1993) Molecular cloning of a complementary DNA encoding a prostate-specific membrane antigen Cancer Res 53(2) 227-30 PMID: 8417812

44. Silver DA et al (1997) Prostate-specific membrane antigen expression in normal and malignant human tissues Clin Cancer Res 3(1) 81-5 PMID: 9815541

45. Sokoloff $\mathrm{R}$ et al (2000) A dual-monoclonal sandwich assay for prostate-specific membrane antigen: levels in tissues, seminal fluid and urine Prostate 43(2) 150-7 PMID: 10754531

46. Kusumi T et al (2008) Immunohistochemical detection of carcinoma in radical prostatectomy specimens following hormone therapy Pathol Int 58(11) 687-94 DOI: 10.1111/j.1440-1827.2008.02294.x PMID: 18844933 
47. Chang SS et al (2001) Comparison of anti-prostate-specific membrane antigen antibodies and other immunomarkers in metastatic prostate carcinoma Urology 57(6) 1179-83 DOI: 10.1016/S0090-4295(01)00983-9 PMID: 11377343

48. Wright GL et al (1996) Upregulation of prostate-specific membrane antigen after androgen-deprivation therapy Urology 48(2) 326-34 DOI: 10.1016/S0090-4295(96)00184-7 PMID: 8753752

49. Perner $\mathrm{S}$ et al (2007) Prostate-specific membrane antigen expression as a predictor of prostate cancer progression Hum Pathol 38(5) 696-701 Epub 2007 Feb 22 DOI: 10.1016/j.humpath.2006.11.012 PMID: 17320151

50. Afshar-Oromieh A et al (2014) Comparison of PET imaging with a (68) Ga-labelled PSMA ligand and (18) F-choline-based PET/ CT for the diagnosis of recurrent prostate cancer Eur J Nucl Med Mol Imaging 41(1) 11-20 Epub 2013 Sep 27 DOI: 10.10071 s00259-013-2525-5 PMID: 24072344 PMCID: $\underline{3843747}$

51. Eiber $\mathrm{M}$ et al (2015) Evaluation of Hybrid ${ }^{68} \mathrm{Ga}$-PSMA Ligand PET/CT in 248 Patients with Biochemical Recurrence after radical prostatectomy J Nucl Med 56(5) 668-74 Epub 2015 Mar 19 DOI: 10.2967/jnumed.115.154153 PMID: 25791990

52. Winter A, Henke RP and Wawroschek F (2015) Targeted salvage lymphadenectomy in patients treated with radical prostatectomy with biochemical recurrence: complete biochemical response without adjuvant therapy in patients with low volume lymph node recurrence over a long-term follow-up BMC Urol 1510 DOI: 10.1186/s12894-015-0004-y PMID: 25881245 PMCID: $\underline{4344760}$

53. Abdollah $\mathrm{F}$ et al (2015) Contemporary role of salvage lymphadenectomy in patients with recurrence following radical prostatectomy Eur Urol 67(5) 839-49 Epub 2014 Mar 26 DOI: 10.1016/j.eururo.2014.03.019 PMID: 24698524 